\title{
Lowering of surface melting temperature in atomic clusters with a nearly closed shell structure
}

\author{
A. Bagrets, ${ }^{1}$ R. Werner, ${ }^{2, *}$ F. Evers, ${ }^{2,1}$ G. Schneider, ${ }^{2, \dagger}$ D. Schooss, ${ }^{1}$ and P. Wölfle ${ }^{2,1}$ \\ ${ }^{1}$ Institut für Nanotechnologie, Forschungszentrum Karlsruhe, D-76021 Karlsruhe, Germany \\ ${ }^{2}$ Institut für Theorie der Kondensierten Materie, Universität Karlsruhe, D-76128 Karlsruhe, Germany
}

(Received 22 June 2009; published 24 February 2010)

\begin{abstract}
We investigate the interplay of particle number, $N$, and structural properties of selected clusters with $N$ $=12$ up to $N=562$ by employing Gupta potentials parameterized for Aluminum and extensive Monte Carlo simulations. Our analysis focuses on closed shell structures with extra atoms. The latter can put the cluster under a significant stress and we argue that typically such a strained system exhibits a reduced energy barrier for (surface) diffusion of cluster atoms. Consequently, also its surface melting temperature, $T_{\mathrm{S}}$, is reduced, so that $T_{\mathrm{S}}$ separates from and actually falls well below the bulk value. The proposed mechanism may be responsible for the suppression of the surface melting temperature observed in recent experiments.
\end{abstract}

DOI: 10.1103/PhysRevB.81.075435

PACS number(s): $36.40 . \mathrm{Ei}, 61.46 . \mathrm{Bc}$

\section{INTRODUCTION}

The properties of small metal clusters have enjoyed considerable interest in recent years. Cluster properties can differ enormously from those of the bulk material due to the large surface-to-volume ratio and due to a remarkable sensitivity of the electronic structure to the cluster size and geometry. ${ }^{1}$ These properties are of interest in technological applications, e.g., for catalysis. ${ }^{2}$ From a conceptual point of view clusters pose fundamental questions in statistical mechanics of finite systems. ${ }^{3}$

The melting process of small clusters is a complex phenomenon, the detailed rules of which are poorly understood. From early on it has been associated with isomer fluctuations. $^{4,5}$ More recent investigations of isolated $\mathrm{Ni}_{13-x} \mathrm{Al}_{x}$ alloy clusters ${ }^{6}$ elucidate the relation between isomer fluctuations and the increase of entropy across the melting transition. A detailed overview about how the phase space grows with increasing particle number and the classification of isomers in terms of potential-energy surfaces is given in Ref. 7. A general overview of structural properties of nanoclusters is found in Ref. 8.

By contrast, the understanding of the melting of very large clusters and bulk materials is considerably better developed. It is believed to be a strongly inhomogeneous process. In large, free metal clusters melting starts in the outermost atom shell, at the interface to the vacuum, because there the thermal fluctuations of the particle density have the lowest energy cost. ${ }^{9,10}$ By feeding more heat into the cluster melting peals off shells from the solid cluster core, layer by layer. Following this logic, one concludes that melting in principle is a continuous transition-to the extent that each one of the individual layers carries its own melting temperature. For atomic clusters this is the rule rather than the exception because typically each layer has its own atomistic structure. This behavior is reflected in the temperature dependence of the specific heat, $C(T)$, which is not necessarily a very sharply peaked function of temperature (as is the case for bulk samples), but rather may exhibit a strong inhomogeneous broadening which reflects intershell averaging. A historical overview on "continuous melting" is given in Ref. 11.

Depending on the crystal orientation, ${ }^{12}$ the surface may melt already at temperatures well below the bulk melting point. ${ }^{13}$ The thickness of the molten layer is strongly temperature dependent. It increases continuously with increasing temperature and eventually it diverges - by definition - at the bulk melting temperature. (It is assumed here, that the thermodynamic state of the cluster interior is unique, i.e., it becomes independent of geometry in the thermodynamic limit.) These observations can be treated theoretically in model calculations using different effective potentials ${ }^{14,15}$ as well as phenomenologically. ${ }^{15}$

Coming back to small systems, this dependency of melting on surface crystallography suggests that surface melting phenomena in atomic clusters should exhibit pronounced size effects, i.e., the melting behavior of two clusters, that differ in size only by one atom, can vary significantly. In particular, the (atomic) structure of closed (atom) shell clusters (magic clusters) is very sensitive to the addition of adatoms or vacancies. ${ }^{16-20}$

Our paper offers a systematic study of selected $\mathrm{Al}$ clusters in a range $12 \leq N \leq 562$ near their melting transition. Here, $N$ is small enough so that a simple extrapolation based on a continuum theory is not applicable and new physics should emerge. Our most crucial observation formulated in general terms: consider splitting the free energy of an $\mathrm{N}$-atom cluster into a bulk and a surface contribution,

$$
F(T, N)=N f_{\mathrm{B}}(T)+N^{2 / 3} f_{\mathrm{S}}(T) .
$$

Both terms, $f_{\mathrm{B}, \mathrm{S}}$, depend on geometrical details of the cluster, i. e. we expect them to become strictly independent of $N$ only in the limit $N \rightarrow \infty$. Formally, $f_{\mathrm{B}, \mathrm{S}}$ are related to two reservoirs, called surface and bulk, with their own specific free energies. The reservoirs are coupled in the sense of the grand canonical ensemble, so they can exchange energy and particles. From this point of view, there is no reason why surface and bulk should have the same, or even a similar, melting temperature. ${ }^{21} \mathrm{~A}$ reason why in metal clusters both temperatures tend to be strongly correlated with one another, nevertheless, is that the interatomic forces at the surface and inside the bulk are similar.

Based on Monte Carlo simulations employing semiempirical Gupta potentials, we propose a general mechanism that can lead to a considerable splitting of the surface and bulk 
melting temperatures. Consider a closed shell cluster, e. g. $\mathrm{Al}_{13}$ or $\mathrm{Al}_{55}$, with icosahedral symmetry. The outer shell of the $\mathrm{Ih}_{55}$ can accommodate an additional atom, an "impurity interstitial," by replacing the fivefold ring structure surounding an edge atom by a sixfold rosettelike ring. The formation of rosettelike structural excitations has been introduced already as a route to amorphisation of $\mathrm{Ih}_{55}$ systems, ${ }^{49}$ and we propose that is relevant for the binding of adatoms in $\mathrm{Ih}_{56}$ and as well.

The impurity is mobile at the surface and its motion strongly assists surface melting. This is, because the atoms inside the meandering deformation field are pushed away from their favorite high-symmetry low-energy site into a more shallow potential well at intermediate position. Our explicit calculations strongly suggest, that by this mechanism the self diffusion of surface atoms can be dramatically enhanced indicating a significant reduction in the activation barrier for diffusion and similarly also of the surface melting temperature. Since impurities do not enter the next (second one counted from outside to inside) cluster shell, there is no corresponding reduction there, so that only two different melting transitions should be discriminated.

This effect may have been seen in two recent experiments. Haberland et al. ${ }^{22,23}$ determined the latent heat and the melting entropy of sodium clusters, $\mathrm{Na}_{N}$, with $N$ $\approx 50-360$. Their modeling of the data provides an excellent phenomenological description assuming the premelting of the cluster surface for nonmagical $N$ values. The microscopic mechanism, that is responsible for this lowering of the surfaces melting temperature, remained unspecified, however. In subsequent theoretical work the experimental melting temperatures have been reproduced quantitatively for a selected set of clusters. ${ }^{25}$ Moreover, the microscopics of premelting of submagic clusters has been already understood in terms of the diffusion of vacancies. ${ }^{24,26}$ Still, the effect of adatoms has not been explicitly analyzed, and the relation to elasticity theory (strain) remained unexplored.

Also, the specific heat of Aluminum cluster cations has been measured recently in the interval $49 \leq N \leq 63$ by multicollision induced dissociation. ${ }^{27}$ Interestingly, the specificheat data for a number of clusters shows signatures of multiple transitions, which have tentatively been interpreted as solid-liquid transitions at the surface that occur below the onset of melting. ${ }^{28}$

\section{METHOD}

To calculate the thermodynamics of an $N$-atom metal cluster, we employ a MC simulation in the canonical ensemble. Technical aspects of our procedure are described in detail in Ref. 29; here, we focus on basic conceptual issues in order to provide the prerequisites necessary for a careful discussion of the numerical observables in Secs. III and IV.

The potential energy of the metal cluster can efficiently be modeled by effective many-body potentials. We shall employ the Gupta potential (GP), ${ }^{30}$ which can be derived in the second moment approximation from a tight-binding model ${ }^{31,32}$ and which correctly describes the surface contraction observed in metals,
TABLE I. Nomenclature of relevant temperature scales of MC simulation with observation time $\tau$ and the corresponding activation energies.

\begin{tabular}{ccc}
\hline \hline$T_{\delta}$ & Onset of intralayer diffusion observed within $\tau$ & $\Delta$ \\
$T_{\mathrm{D}}$ & Onset of interlayer diffusion observed within $\tau$ & $\Delta_{i o}$ \\
$T_{\mathrm{C}}$ & Maximum of specific heat \\
\hline \hline
\end{tabular}

$$
V\left(\left\{r_{i j}\right\}\right)=\sum_{i}^{N}\left[\sum_{j \neq i}^{N} A e^{-p \bar{r}_{i j}}-\sqrt{\sum_{j \neq i} \xi^{2} e^{-2 q \bar{r}_{i j}}}\right] .
$$

Here, $i$ and $j$ are atom labels, $\bar{r}_{i j}=r_{i j} / r_{0}-1$, and $r_{i j}=\left|\boldsymbol{r}_{i}-\boldsymbol{r}_{j}\right|$ is the modulus of the distance between two atoms at positions $\boldsymbol{r}_{i}$ and $\boldsymbol{r}_{j}$. The parameters have been determined by fitting the experimental bulk lattice parameters and elastic moduli ${ }^{33}$ as $A=0.1221 \mathrm{eV}, \xi=1.316 \mathrm{eV}, p=8.612$, and $q=2.516$ for $\mathrm{Al}$. Distances are measured in units of the bulk first neighbor distance $r_{0}=2.864 \AA$. A standard Metropolis algorithm is employed ${ }^{29,34,35}$ with boundary conditions imposed by a hard wall cube with linear dimension $L$ : a shift of a single atom by a randomly chosen vector with a length taken from the interval $\left[0, \kappa(T) r_{0}\right]$ is offered with an associated change of the cluster energy $\Delta E$. A temperature, $T$, is introduced via the probability, $p$, to accept such a step with $p \sim \exp \left(-\Delta E / k_{\mathrm{B}} T\right)$; for the most important temperature scales, see Table I. The parameter $0<\kappa(T)<1$ is chosen so that the acceptance rate is close to $50 \%$; a typical value at intermediate temperatures is $\kappa=0.25$. The cluster is updated after each accepted move. Runs are performed with sampling rates of up to $8 \times 10^{7}$ steps per temperature and atom.

\section{A. Observables}

In order to characterize the thermodynamic state of the cluster we introduce the following observables:

(i) the specific heat can be obtained from the ensemble averages of the potential energy $V$ and its square,

$$
\frac{C}{k_{\mathrm{B}}}=\frac{1}{N k_{\mathrm{B}}^{2} T^{2}}\left(\left\langle V^{2}\right\rangle-\langle V\rangle^{2}\right)+\frac{3}{2} .
$$

The kinetic contribution $C_{\text {kin }}=3 / 2 k_{\mathrm{B}}$ per atom has been added here. (Note that we treat the metal cluster as a gas of distinguishable particles rather than indistinguishable ones. For the specific heat, a double-counting problem does not arise, since it is a second derivative of the free energy.)

Invoking ergodicity, in practical calculations the ensemble average $\langle\ldots\rangle$ is frequently combined with (or even replaced by) an average over the MC "time," $\tau$, i.e., the total number of MC steps,

$$
\left\{\mathcal{O}_{i j}\right\}(\tau)=\frac{1}{\tau} \sum_{n=1} \mathcal{O}_{i j}^{(n)},
$$

counting is started after equilibrating an initial configuration. The two-body term $\mathcal{O}$ takes a value $\mathcal{O}^{(n)}$ in the $n$th MC step.

(ii) One may also introduce the rms pair index 


$$
d_{i j}(\tau)=\sqrt{\left\{r_{i j}^{2}\right\}-\left\{r_{i j}\right\}^{2}} /\left\{r_{i j}\right\}
$$

to study the MC time evolution. The interest in this quantity stems from the following very general observation: Consider a configuration space for an $N$-body system which has the property that several regions exist where the free energy takes (local) minima. After a transient time interval ("warm up" or partial equilibration period) the MC dynamics starts to explore one of these minima. There will be a typical time scale (corresponding to an activation energy) involved, after which the entire $\mathrm{N}$-body system migrates to a second, competing minimum where the procedure repeats itself. Thus, the MC dynamics allows to study aspects of the energy landscape associated with the configurational space, such as activation barriers. What has been described here for a general $\mathrm{N}$-body system remains equally valid for the pair of two particles, Eq. (5), embedded in an environment consisting of $N-2$ other particles.

In the limit $\tau \rightarrow \infty$ the evolution in MC "time" is ergodic, so the $\mathrm{N}$-atom cluster will explore all the phase space available. This implies, that at any temperature $T>0$ cluster atoms are deconfined: there is a finite time after which the $i$-atom has migrated from its initial position into any other given cluster site. Therefore, $d_{i j}$ takes the same value, $d_{\mathrm{B}}$ for any given pair of atoms and is a unique function of the temperature and the particle number: $d_{\mathrm{B}}(T, N)$. Since all diffusion processes terminate at the cluster size independent of the diffusion constant (i.e., temperature), $d_{\mathrm{B}}(T, N)$ has only a weak $T$ dependence about a mean value, that incorporates crude information about the overall cluster geometry (spherical vs quasi-one-dimensional), but nothing else. It is implied that the limit $d_{\mathrm{B}}$ is usually not very sensitive to the melting transition.

For averages like the one defined in Eq. (5) the ergodicity theorem strictly holds only at $\tau \rightarrow \infty$; at any finite $\tau$ the value for $d_{i j}(\tau)$ can vary between the different pairs of atoms $i, j$. If it so happens, that $n$ different classes of pairs exist, where each class just samples its own local minimum in-phase space within $\tau$, then $d_{i j}(T)$ can develop $n$ branches.

Quite generally, in situations where different pairs sample different sectors of phase space, the convergence with $\tau$ can be increased by averaging over all the different pairs. In this spirit, we define one more average

$$
\delta_{\mathrm{B}}(\tau)=\frac{1}{N(N-1)} \sum_{i, j \neq i} d_{i j}(\tau) .
$$

which we will refer to as the "Berry parameter." ${ }^{36}$ It has a more rapid convergence behavior, $\delta_{\mathrm{B}}(\tau) \rightarrow d_{\mathrm{B}}$, and therefore is easier to investigate in numerical simulations than the rms pair index.

\section{B. General properties of $\delta_{\mathrm{B}}$ and the rms pair index $d_{i j}$}

In order to illustrate the general properties of Berry parameter and pair index, we now consider as an example $\mathrm{Al}_{12}$, $\mathrm{Al}_{13}$, and $\mathrm{Al}_{14}$ (Fig. 1). We display the Berry parameter, Eq. (6), in Figs. 1(d)-1(f) for clusters with $N=12,13,14$.

At smallest temperatures only thermal vibrations of the atoms around a single site are observed within the MC win-

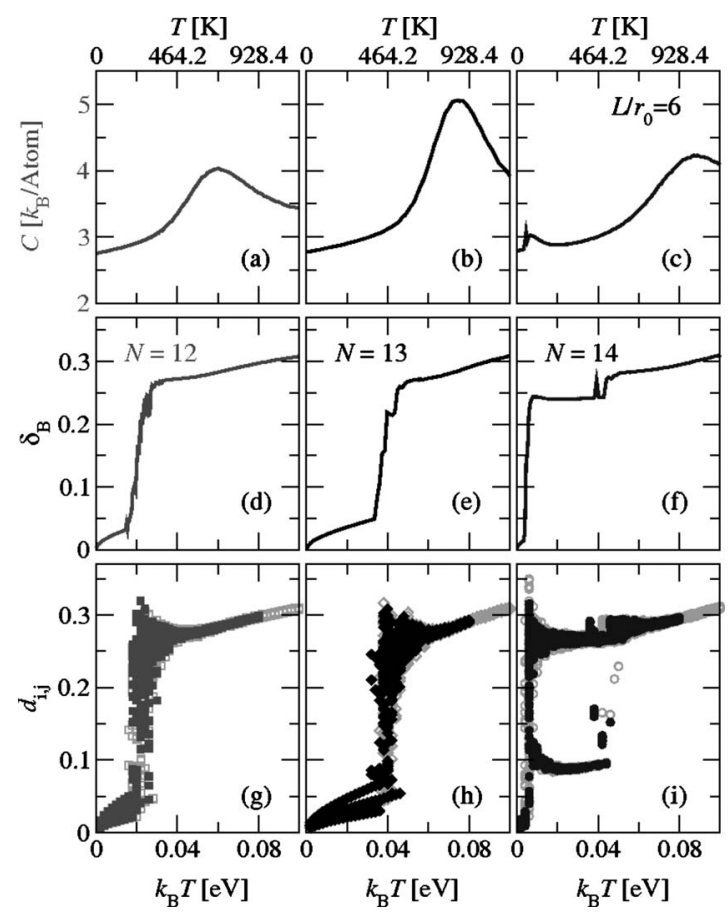

FIG. 1. Characteristic temperatures for clusters of size (a), (d), and (g) $N=12$, (b), (e), and (h) $N=13$, and (c), (f), and (i) $N=14$. Panels (a)-(c) show the specific heat, panels (d)-(f) the Berry parameter, while panels (g)-(i) show the individual rms bond-length fluctuations from Eq. (5). Panels (a)-(f) show data for $\tau=8 \times 10^{7}$. In panels (g)-(i) dark symbols correspond to $8 \times 10^{7} \mathrm{MC}$ steps while gray points are obtained with $4 \times 10^{7} \mathrm{MC}$ steps per atom.

dow of time. Hence, the Berry parameter grows $\propto \sqrt{T}$ reflecting the virial theorem applied to the harmonic oscillator. This asymptotic low-temperature behavior is clearly observed in the traces Fig. 1.

Also easily understood is the behavior at temperatures higher than $T_{\delta}$, at which the Berry parameter exhibits a very sharp jump. An estimate of $\delta_{\mathrm{B}}$ in this regime may be obtained, by taking the ground-state geometry and calculating the average squared displacements by assuming ergodicity, i.e., that in the $\mathrm{MC}$ time evolution each occupation of allowed sites occurs with the same probability. For example, in the case of $\mathrm{Al}_{13}$ one thus finds $\delta_{\mathrm{B}} \approx 0.25$ which agrees reasonably well with the data (Fig. 1).

The sharp increase in $\delta_{\mathrm{B}}$ at the intermediate temperature, $T_{\delta}$, signalizes the onset of cyclic, correlated exchanges of (surface) atoms between their various positions. (The weak irregularities, which are still visible, resemble residual statistical noise.) In the spirit of our earlier discussion, we do not expect that $T_{\delta}$ is independent of our observation time $\tau{ }^{37} \mathrm{In}$ fact, the precise meaning of $T_{\delta}$ is the following: at $T_{\delta}$ the observation time $\tau$ has been long enough, so that at $T>T_{\delta}$ processes can be observed where atoms trade places with one another even though the probability $p$ for this to happen may be exponentially suppressed with a factor $\exp \left(-\Delta / k_{\mathrm{B}} T\right) . \Delta$ denotes the corresponding activation energy which will in general exhibit a weak (i.e., nonsingular) temperature dependency.

Our argument shows that $T_{\delta}$ itself cannot immediately be identified with any intrinsic energy scale of the free cluster, 


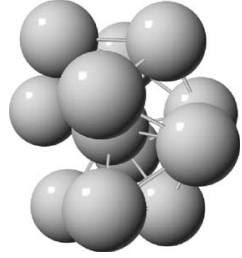

(a)

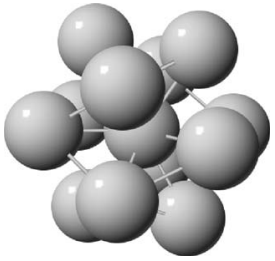

(b)

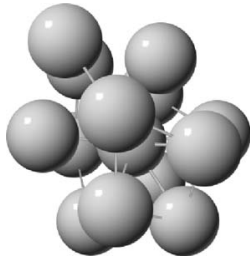

(c)
FIG. 2. Ground-state configurations of $\mathrm{Al}_{12}$ (left), $\mathrm{Al}_{13}$ (center), and $\mathrm{Al}_{14}$ (right) as obtained with Gupta potentials. The hole has little impact on the position of the remaining atoms. By contrast, the adatom creates a docking site with a higher coordination number, which is incompatible with the threefold symmetries of the docking sites of the unperturbed $\mathrm{Al}_{13}$. Hence, $\mathrm{Al}_{14}$ experiences significant strain.

such as a surface melting temperature. The specific heat peaks only at a much higher temperature, $T_{\mathrm{C}} \gg T_{\delta}$, which indicates the volume melting temperature of the cluster [see Figs. 1(a)-1(c)].

It is possible to obtain an estimate of the activation energy $\Delta$ from the way that $T_{\delta}$ flows with the observation time $\tau$. Namely, one has $\Delta^{-1} \sim d T_{\delta}^{-1} / d \ln (\tau)$. Unfortunately, in order to obtain very accurate scaling with $\ln (\tau)$ the calculational effort goes well beyond what was achievable within this study.

Further information is carried by the pair index $d_{i j}$, which is displayed in Figs. 1(g)-1(i) at two values of $\tau$. At $N=13$, a three branch structure is readily identified at $T<T_{\delta}$. The branches reflect the fact, that three kinds of atom pairs exist (center atom/shell atom, shell neighbors, shell next-nearest neighbors), that have different distance fluctuations, see Fig. 2. Two branches are found with $N=14$ even at $T>T_{\delta}$. The upper one stems from the on-shell pairings while the lower branch represents the mixed pairs, center atom/shell atom. Consistent with this picture, the lower branch contains 13 bonds, which are the 13 bonds between the center atom and the 13 statistically equivalent surface atoms. This latter branch exists only in the intermediate temperature interval $T_{\delta}<T<T_{\mathrm{D}}$. The role of the temperature $T_{\mathrm{D}}$ is similar to $T_{\delta}$, except that the associated activation energy, $\Delta_{i o}$, now is related to an exchange of inner atoms with the outer shell. At $T_{\mathrm{D}}$ the Berry parameter, $\delta_{\mathrm{B}}$, exhibits a second sharp increase. The discussion of the pair index allows us to attribute this increase as being due to the center atom now being deconfined within the $\mathrm{MC}$ window of time.

\section{SELF-DIFFUSION}

The influence of closed shells on the cohesive energies of metal clusters ${ }^{7,16-18,29}$ in the gas phase and their melting points ${ }^{35,38}$ has been a focus of research for quite some time. In this section we use the Berry parameter to investigate the onset of self diffusion of atoms within such systems.

\section{A. $\mathbf{A l}_{14}$}

The low-temperature jump in the Berry parameter for $\mathrm{Al}_{14}$ in Fig. 1 has been identified previously as induced by the
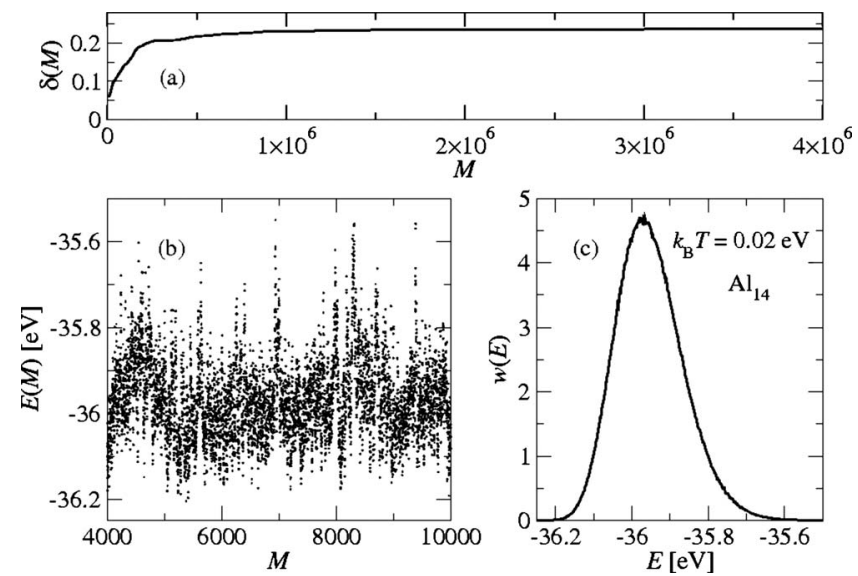

FIG. 3. Potential-energy statistics for $\mathrm{Al}_{14}$ at $k_{\mathrm{B}} T=0.02 \mathrm{eV}$ in the surface molten phase. (a) Convergence of the Berry parameter as a function of MC steps. (b) Sample of the potential energies of accepted configurations during the same MC run as in (a). (c) Histogram of the potential-energy distribution function $w(E)(2.4$ $\times 10^{6}$ energy values, 480 bins).

temporary absorption of the adatom on the $\mathrm{Al}_{13}$ icosahedral core structure into the surface (see Fig. 2). ${ }^{29}$ Here we witness the effect, that has been described in general terms already in the introduction. The adatom destabilizes the high-symmetry surface of the $\mathrm{Al}_{13}$ cluster and therefore the activation barrier for self diffusion on the surface is reduced, by a factor of roughly $T_{\delta}\left(\mathrm{Al}_{14}\right) / T_{\delta}\left(\mathrm{Al}_{13}\right) \sim 5$ according to our calculation.

In order to obtain a more detailed understanding of the thermodynamics of the system, in Fig. 1(i) the temperature dependence of the rms pair index is shown. In particular, we observe that $T_{\mathrm{D}}\left(\mathrm{Al}_{14}\right) \gtrsim T_{\delta}\left(\mathrm{Al}_{13}\right)$. This suggests, that the core atom of $\mathrm{Al}_{14}$ must overcome a slightly increased barrier (as compared to $\mathrm{Al}_{13}$ ) to enter the (strained) outer shell.

For illustration, the potential-energy statistics for $\mathrm{Al}_{14}$ are shown in Fig. 3 at an intermediate temperature $\left(k_{\mathrm{B}} T\right.$ $=0.02 \mathrm{eV}$ ), where the pair index exhibits two branches (Fig. 1). Panel (a) shows the convergence behavior of the Berry parameter as a function of $\tau$. Panel (b) displays potential energies of accepted configurations during the sample MC run. Panel (c) shows the potential-energy distribution function, $w(E)$. Consistent with our interpretation of the branching behavior and with earlier results for $\mathrm{Ni}_{14}$ the distribution shows no sign of phase coexistence. ${ }^{21,39,40}$

The results presented here for $\mathrm{Al}_{14}$ are analogous to those for $\mathrm{Pb}_{14}$ with very similar characteristic temperatures. ${ }^{41}$ They differ from $\mathrm{Ni}_{14}$ (Ref. 42) and $\mathrm{Cu}_{14}$ (Ref. 48) in so far, as for $\mathrm{Al}_{14}$ no adatom hopping is observed.

The clusters with $15 \leq N \leq 18$ show a behavior similar to $\mathrm{Al}_{14}$. Apparently, the presence of several additional adatoms has qualitatively a similar (destabilizing) effect on the surface as a single adatom. Panels (a)-(c) of Fig. 4 show the individual bond-length fluctuations $\mathrm{Eq}$. (5) for $\mathrm{Al}_{15-17}$, respectively. For $\mathrm{Al}_{18}$ (not shown here, c.f. Fig. 7) similar results are obtained.

\section{B. $\mathrm{Al}_{56}$ and $\mathrm{Al}_{57}$}

In order to see whether the lowering of activation barriers for surface diffusion may indeed be a typical phenomenon 


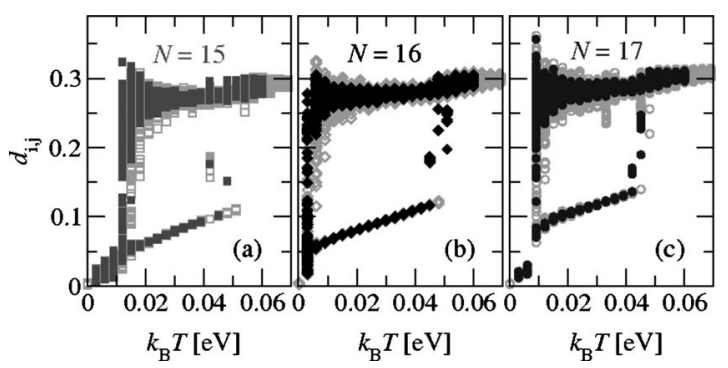

FIG. 4. Temperature dependence of the individual bond fluctuations Eq. (5) for clusters of size (a) $N=15$, (b) $N=16$, and (c) $N$ $=17$. The "solid" bonds with $d_{i j} \leq 0.1$ correspond to those between the central atom and the surface atoms. In the surface molten phase all surface atoms are equivalent. Dark symbols correspond to 8 $\times 10^{7}$ MC steps while gray open symbols are obtained with 2 $\times 10^{7}$ MC steps per atom.

for closed shell configuration with one excess atom, we now investigate the case $\mathrm{Al}_{56}$.

Figure 6 shows the specific heat, the Berry parameter and the pair index for $N=55,56$, and 57 , respectively. The dramatic suppression of $T_{\delta}$ in $\mathrm{Al}_{56}$ seen in $\delta_{\mathrm{B}}$ as compared to the closed shell case $\mathrm{Al}_{55}$ was already observed before. ${ }^{29}$

To obtain more detailed information, we consider the pair index Fig. 6(i). The lower branch at $T_{\delta}<T<T_{\mathrm{D}}$ represents 331 pairs. They correspond to all pairings within the set of atoms that consists of the $\mathrm{Al}_{13}$ core together with the 12 outer corner atoms of the (distorted) icosahedron-subset of confined atoms. The remaining 31 surface atoms are deconfined. The upper branch represents the cross pairings between the two sets of atoms as well as the pairings between deconfined atoms. The data suggest that edge atoms of surface facets are more mobile than the corner atoms.

In order to understand the reason for this enhanced mobility, we display in Fig. 5 the (Gupta-potential-based) ground-state structures of $\mathrm{Al}_{56}$ and $\mathrm{Al}_{55}$. The large full circles indicate the 12 corner atoms, which move very little at $T_{\delta}$ $<T<T_{\mathrm{D}}$. It is seen, that the adatom integrates by promoting the environment of one of the corner atoms from its original fivefold symmetry to an (approximate) local sixfold symmetry. This implies a sizable bond-length mismatch of about $1 / 5 \sim 20 \%$, that creates a deformation field. Now, if an atom out of the six ring pushes away another atom to enter a new neighboring facet, then the entire deformation field will follow. Actually, what really diffuses over the surface is the (a)

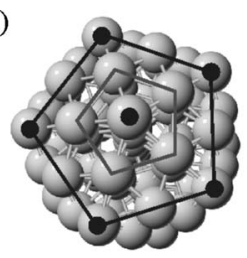

(b)

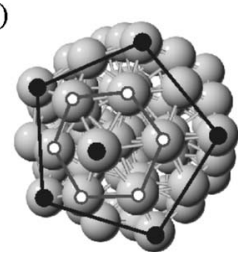

FIG. 5. Ground-state configurations of (a) $\mathrm{Al}_{55}$ and (b) $\mathrm{Al}_{56}$ as obtained with Gupta potentials. Large full circles indicate the corner atoms, which remain "solid" in the partially surface molten state of $\mathrm{Al}_{56}$. Small open circles [rosette structure (Ref. 49)] show the broken fivefold symmetry caused be the 56th atom absorbed into the surface of the icosahedron.

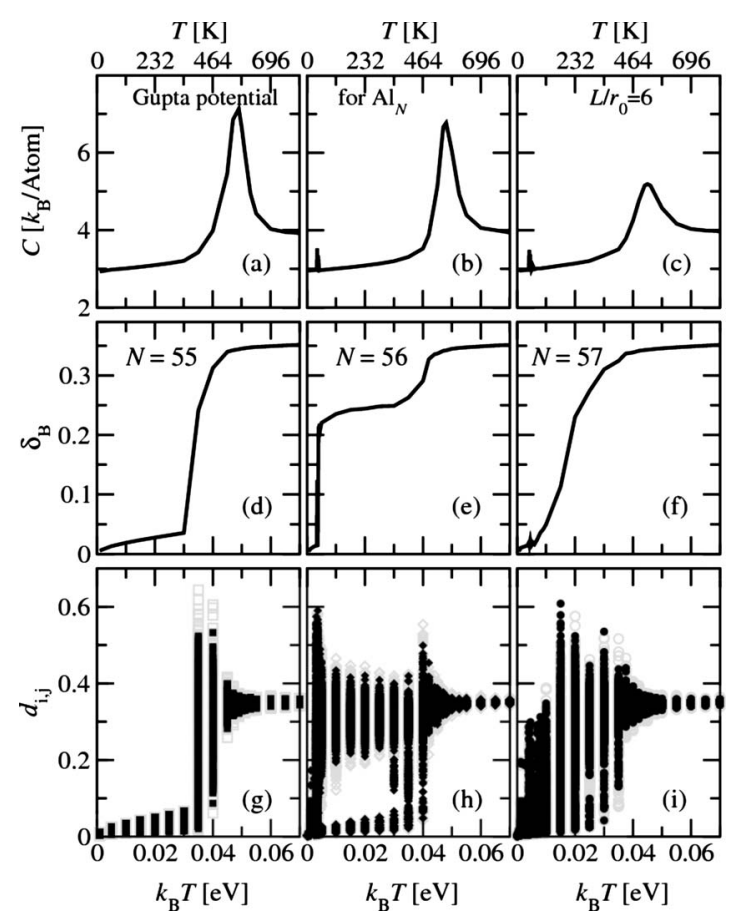

FIG. 6. Temperature dependence of the melting transition for clusters of size (a), (d), and (g) $N=55$, (b), (e), and (h) $N=56$, and (c), (f), and (i) $N=57$. Panels (a)-(c) show the specific heat, panels (d)-(f) the Berry parameter, while panels (g)-(i) show the individual rms bond-length fluctuations from Eq. (5). Panels (a)-(d) show data for $8 \times 10^{7} \mathrm{MC}$ steps per atom; in panels (g)-(i) dark symbols correspond to $8 \times 10^{7} \mathrm{MC}$ steps while gray open symbols are obtained with $4 \times 10^{7} \mathrm{MC}$ steps per atom.

sixfold rosette structure (Fig. 5). The energy barrier to be overcome in this self diffusion process is relatively low. This is because it is not necessary to first create a hole in the crystal lattice of the target facet for the rosette to move there.

According to the scenario developed for $\mathrm{Al}_{56}$, two rosettes should decorate the surface of $\mathrm{Al}_{57}$. A naive expectation is that these rosettes repel each another, because it is more difficult for a second rosette to enter an area that is under strain already from the presence of a first one. Since the two rosettes cover a large surface fraction of $\mathrm{Al}_{57}$, diffusion barriers should be increased again as compared to the "free" case $\mathrm{Al}_{56}$. Indeed, as can be seen in Fig. 6, the sharp increase observed for $\mathrm{Al}_{56}$ at very low temperatures in the Berry parameter almost disappears for $\mathrm{Al}_{57}$. It gives way to a very broad shoulder which is indicative of a large number of energy scales that is associated with a strongly disturbed ("disordered") outer cluster shell.

Experiments. In a recent experiment, a broad peak in the specific heat, $C(T)$, of $\mathrm{Al}_{56}^{+}$and $\mathrm{Al}_{57}^{+}$has been measured and analyzed. ${ }^{27,28}$ The authors were concluding, that $\mathrm{Al}_{56}^{+}$undergoes a separate transition even before the entire cluster starts to melt. The physical nature of the first transition could not be clearly resolved. It would be tempting to propose that our research supports the supposition of the authors that premelting of the surface is a possible candidate. Namely, since low diffusion barriers are usually also indicative of low melting temperatures, our scenario would suggest that premelting of the surface may occur well before melting of the bulk in 
$\mathrm{Al}_{56}^{+}$. However, such a direct application of our ideas to experiments ${ }^{27,28}$ is not without difficulty and probably not indicated: there is a trend in the theoretical data, Fig. 6, that the latent heat (integral under the peak in the specific heat) decreases from $N=55$ to $N=57$. This is plausible, because it takes less energy per atom to melt a structure under strain. By contrast, the experimental trend is reverse and the latent heat increases for the series $N=55,56,57$. The likely reason for this discrepancy is that Gupta potentials cannot give a sufficiently realistic description of the thermodynamics of aluminum clusters, $\mathrm{Al}_{N}^{+},(N=55,56,57)$ near the melting transition. That indeed difficulties exist even with the uncharged species, $\mathrm{Al}_{N}$, is signalized by explicit density functional theory calculations, which show that the $T=0$ groundstate conformation of $\mathrm{Al}_{55}$ is not icosahedral ${ }^{17,19}$ contradicting what is found with Gupta potentials. This casts a doubt on the applicability of Gupta potentials to the specific metal aluminum for simulations of low-temperature behavior. However, we would like to emphasize that our general findings remain valid for other materials with an isocahedral ground state as well as for those situations, where an approximate icosahedral symmetry is restored at slightly higher temperatures.

\section{CHARACTERISTIC TEMPERATURES FOR $\mathrm{Al}_{N}$ CLUSTERS}

The delicate interplay between the special geometry of $\mathrm{Al}_{55}$ and the low activation barrier for adatom diffusion in $\mathrm{Al}_{56}$ and $\mathrm{Al}_{57}$ does not prompt the expectation, that this specific mechanism is ubiquitous in all its details. Nevertheless, there is a lesson to be learned about the more general case. One expects a lowering of the activation energy for surface diffusion of surface atoms of clusters with structures that derive from high-symmetry parent states either by (i) punching in vacancies or (ii) by inserting adatoms into its outer shell. In such systems, there is a possibility for atomic motions, which are not just ring exchanges and which also do not require to break chemical bonds to first create a vacancy. Since low diffusion barriers are also indicative of low melting temperatures, here is a mechanism by which surface melting may become a process that should be distinguished, in principle, from the melting of the bulk.

This analysis suggests that the ratio $T_{\delta} / T_{\mathrm{D}}$ tends to be large for structures with closed shells or subshells and much smaller otherwise. We have tested this idea by calculating $T_{\mathrm{C}}$, the temperature at which the specific heat $C(T)$ takes its maximum, $T_{\delta}$ and $T_{\mathrm{D}}$ (at fixed $\tau$ ) for a variety of different cluster sizes. Figure 7 comprises our results which, we believe, support our general picture: the ratio of $T_{\delta} / T_{\mathrm{D}}$ takes peak values at closed shell structures and much lower ones almost everywhere else (except for the smallest cluster sizes, where our previous analysis does not apply).

We mention that the $\mathrm{Al}_{N}$ clusters with $14 \leq N \leq 18$ and $N=24$ have maxima of the specific heat at temperatures larger than the $\mathrm{Al}$ bulk melting temperature of $T_{\text {bulk }}$ $=933 \mathrm{~K}=0.0804 \mathrm{eV} / k_{\mathrm{B}}$. This observation is in line with the empirical investigation of the melting of small Sn (Ref. 43) and Ga (Ref. 44) clusters, which have revealed a possible

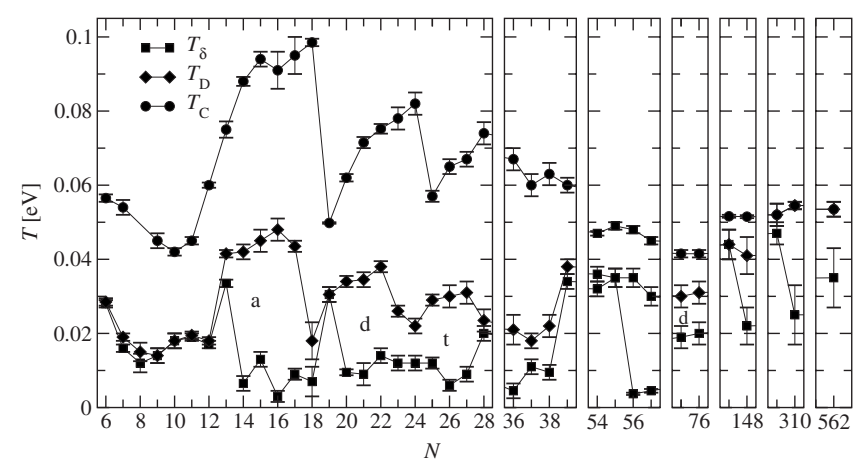

FIG. 7. Characteristic temperatures of $\mathrm{Al}_{N}$ associated with self diffusion over the cluster size $N$; for the definitions of $T_{\delta}, T_{\mathrm{D}}, T_{\mathrm{C}}$ see Table I. "a" labels a "confined" central atom, "d" a "confined" central dimer, and " $t$ " a "confined" central trimer. [The onset of the evaporation transition in the investigated systems is found at larger temperatures $T_{\text {evap }}>0.12 \mathrm{eV}$ (Ref. 29).]

stability of the solid phase of the particles beyond the melting temperature of the bulk material. In these cases the high cluster melting temperature was interpreted as a consequence of the rigidity of the specific ground-state structures of the clusters. This interpretation found support from microcanonical molecular-dynamics (MD) calculations for $\mathrm{C}, \mathrm{Si}, \mathrm{Ge}$, and Sn clusters ${ }^{45}$ as well as for isokinetic MD investigations of $\mathrm{Sn}_{10}$ (Refs. 27 and 46) and $\mathrm{Ga}_{13}, \mathrm{Ga}_{17}$ (Ref. 47) particles.

We briefly touch upon the limit of large clusters, $N$ $\gg 100$. There, we observe that $T_{\mathrm{D}} \rightarrow T_{\mathrm{C}}$, while $T_{\delta}$ does not appear to follow this trend. To understand this behavior, recall that $T_{\mathrm{D}}$ is the temperature at which our MC time has become long enough, so we can observe an exchange of particles between the outer shell and its inner neighbor. Then, $T_{\mathrm{D}} \approx T_{\mathrm{C}}$ implies that intershell exchange cannot be observed-even with our very long observation timesunless we actually heat up the entire cluster to melt. This behavior is consistent with what one would expect for a macroscopic single-crystal grain: as long as the crystalline structure of the surface is intact $\left(T \ll T_{\text {melt }}\right)$ one has $\Delta \ll \Delta_{i o}$ and interlayer diffusion is strongly suppressed. However, after melting the surface layer no longer forces the atoms of its neighboring inner shell into a crystalline structure. Hence, intra self diffusion within the second layer becomes (almost) as cheap energetically as was diffusion in the first layer, before. Therefore, the second layer melts immediately after the first one, so at melting one has for the "effective" activation energies: $\bar{\Delta} \approx \bar{\Delta}_{i o} \sim T_{\text {melt }}$. In other words: we recover the standard "continuous melting" scenario.

\section{CONCLUSION}

In this paper we have argued, that certain metal clusters with an intermediate size may exhibit a property that cannot be found in bulk materials: the activation energies for surface diffusion, $\Delta$, and interlayer diffusion, $\Delta_{i o}$, are substantially different from one another: $\Delta_{i o} \gg \Delta$. Since these activation energies are closely tied to melting temperatures, one expects that the outermost surface layer can exhibit its own melting transition, which is well separated from the bulk. The con- 
tinuous melting of the cluster core should start only at much higher temperatures.

The most dramatic decrease in $\Delta$ has been found with $\mathrm{Al}_{56}$. In this case, it is the "frustration" of atomic bonds that originates from implanting an adatom into a closed shell system, which produces the effect. A related mechanism leads to a decrease in the surface melting temperature also for $\mathrm{Al}_{14}$ and is expected to be active in clusters, where $N$ is slightly above some magical (closed atom shell) value $N^{*}$. We mention that a distortion of the outer cluster shell is also present in clusters with $N$ slightly below $N^{*}$. However, a hole is usually accommodated more easily than an adatom and therefore the decrease tends to be asymmetric: it is typically stronger for $N>N^{*}$ as compared to $N<N^{*}$.

Monatomic, macroscopic and planar metal surfaces, that face the vacuum, do not easily allow for the frustration of surface bonds that we have observed with the metal clusters. An attempt to locally implant concentration of adatoms into macroscopic surfaces beyond a certain threshold would result in a metastable state that eventually would transform into another state without frustration, where the adatoms would have undergone island formation.
On the other hand, our research suggests the design of materials with a surface melting temperature that is strongly diminished and separates from the bulk melting temperature by a controllable amount. The idea is to employ a monatomic core and a biatomic shell structure. The purpose of pressing foreign atoms into the outermost layer of the host material's crystal is to create local strain fields. Since strain reduces the local melting temperature, heating up such a system could create puddles of molten host material on top of the solid, bulk core. Clearly, the combination of host and implantation materials should satisfy at least two conditions: (i) the implantations should have a high solubility in the host material, but (ii) they should not easily diffuse away from the surface into the bulk of the crystal, either. Whether indeed a combination of suitable materials can be found, this we have to leave for future research.

\section{ACKNOWLEDGMENTS}

Instructive discussions with $\mathrm{M}$. Blom and $\mathrm{P}$. Schmitteckert are gratefully acknowledged. The work was supported by the Center for Functional Nanostructures of the Deutsche Forschungsgemeinschaft within Project No. C4.7.
*Present address: DekaBank Deutsche Girozentrale, 60325 Frankfurt, Germany.

†Present address: Department of Physics, Oregon State University, 301 Weniger Hall Corvallis, OR 97331-6507, USA.

${ }^{1}$ H. Häkkinen, S. Abbet, A. Sanchez, U. Heiz, and U. Landman, Angew. Chem., Int. Ed. 42, 1297 (2003).

${ }^{2}$ S. Rojas, S. Eriksson, and M. Boutonnet, Catalysis 17, 258 (2004); P. L. Gai and E. D. Boyes, Electron Microscopy in Heterogeneous Catalysis, Series in Microscopy in Materials Science (Institute of Physics, New York, 2003), Chap. 5.

${ }^{3}$ D. Gross, Microcanonical Thermodynamics: Phase Transitions in "Small" Systems (World Scientific Publishing Co. Pts. Ltd, Singapore, 2001).

${ }^{4}$ F. G. Amar and R. S. Berry, J. Chem. Phys. 85, 5943 (1986).

${ }^{5}$ J. Jellinek, T. L. Beck, and R. S. Berry, J. Chem. Phys. 84, 2783 (1986).

${ }^{6}$ E. B. Krissinel and J. Jellinek, Int. J. Quantum Chem. 62, 185 (1997).

${ }^{7}$ D. J. Wales, J. P. K. Doye, M. A. Miller, P. N. Mortenson, and T. R. Walsh, Adv. Chem. Phys. 115, 1 (2000).

${ }^{8}$ F. Baletto and R. Ferrando, Rev. Mod. Phys. 77, 371 (2005).

${ }^{9}$ R. M. Goodman and G. A. Somorjai, J. Chem. Phys. 52, 6325 (1970).

${ }^{10}$ R. W. Cahn, Nature (London) 323, 668 (1986).

${ }^{11}$ J. G. Dash, Contemp. Phys. 43, 427 (2002).

${ }^{12}$ B. Pluis, A. W. Denier van der Gon, J. W. M. Frenken, and J. F. van der Veen, Phys. Rev. Lett. 59, 2678 (1987).

${ }^{13}$ J. W. M. Frenken and J. F. van der Veen, Phys. Rev. Lett. 54, 134 (1985)

${ }^{14}$ R. Ohnesorge, H. Löwen, and H. Wagner, Phys. Rev. E 50, 4801 (1994).

${ }^{15}$ F. D. DiTolla, F. Ercolessi, and E. Tosatti, Phys. Rev. Lett. 74,
3201 (1995).

${ }^{16}$ J. P. K. Doye and D. J. Wales, New J. Chem. 1998, 733 (1998).

${ }^{17}$ R. Ahlrichs and S. D. Elliot, Phys. Chem. Chem. Phys. 1, 13 (1999).

${ }^{18}$ G. W. Turner, R. L. Johnston, and N. T. Wilson, J. Chem. Phys. 112, 4773 (2000).

${ }^{19}$ A. Aguado and J. M. Lopez, J. Phys. Chem. B 110, 1402 (2006).

${ }^{20}$ E. G. Noya, J. P. K. Doye, D. J. Wales, and A. Aguado, Eur. Phys. J. D 43, 57 (2007).

${ }^{21}$ R. E. Kunz and R. S. Berry, Phys. Rev. Lett. 71, 3987 (1993).

${ }^{22}$ H. Haberland, T. Hippler, J. Donges, O. Kostko, M. Schmidt, and B. von Issendorff, Phys. Rev. Lett. 94, 035701 (2005).

${ }^{23}$ M. Schmidt, R. Kusche, B. V. Issendorff, and H. Haberland, Nature (London) 393, 238 (1998).

${ }^{24}$ A. Aguado, J. Phys. Chem. B 109, 13043 (2005).

${ }^{25}$ A. Aguado and J. M. Lopez, Phys. Rev. Lett. 94, 233401 (2005).

${ }^{26}$ S. Krishnamurty, G. S. Shafai, D. G. Kanhere, B. S. de Bas, and M. J. Ford, J. Phys. Chem. A 111, 10769 (2007).

${ }^{27}$ G. A. Breaux, C. M. Neal, B. Cao, and M. F. Jarrold, Phys. Rev. Lett. 94, 173401 (2005).

${ }^{28}$ C. M. Neal, A. K. Starace, and M. F. Jarrold, Phys. Rev. B 76, 054113 (2007).

${ }^{29}$ R. Werner, Eur. Phys. J. B 43, 47 (2005).

${ }^{30}$ R. P. Gupta, Phys. Rev. B 23, 6265 (1981).

${ }^{31}$ D. Tománek, S. Mukherjee, and K. H. Bennemann, Phys. Rev. B 28, 665 (1983).

${ }^{32}$ W. Zhong, Y. S. Li, and D. Tománek, Phys. Rev. B 44, 13053 (1991).

${ }^{33}$ F. Cleri and V. Rosato, Phys. Rev. B 48, 22 (1993).

${ }^{34}$ M. P. Allen and D. J. Tildesley, Computer Simulation of Liquids, Oxford Science Publications (Clarendon Press, Oxford, 1989).

${ }^{35}$ J. Wang, F. Ding, W. Shen, T. Li, G. Wang, and J. Zhao, Solid 
State Commun. 119, 13 (2001).

${ }^{36}$ Y. Zhou, M. Karplus, K. D. Ball, and R. S. Berry, J. Chem. Phys. 116, 2323 (2002)

${ }^{37}$ D. D. Frantz, J. Chem. Phys. 102, 3747 (1995).

${ }^{38}$ P. Blaise and S. A. Blundell, Phys. Rev. B 63, 235409 (2001).

${ }^{39}$ R. E. Kunz and R. S. Berry, Phys. Rev. E 49, 1895 (1994).

${ }^{40}$ A. Proykova, S. Pisov, and R. Berry, J. Chem. Phys. 115, 8583 (2001).

${ }^{41}$ Y. J. Lee, E.-K. Lee, S. Kim, and R. M. Nieminen, Phys. Rev. Lett. 86, 999 (2001).

${ }^{42}$ Y. J. Lee, J. Y. Maeng, E.-K. Lee, B. Kim, S. Kim, and K.-K. Han, J. Comput. Chem. 21, 380 (2000).

${ }^{43}$ A. A. Shvartsburg and M. F. Jarrold, Phys. Rev. Lett. 85, 2530
(2000).

${ }^{44}$ G. A. Breaux, R. C. Benirschke, T. Sugai, B. S. Kinnear, and M. F. Jarrold, Phys. Rev. Lett. 91, 215508 (2003).

${ }^{45}$ Z.-Y. Lu, C.-Z. Wang, and K.-M. Ho, Phys. Rev. B 61, 2329 (2000).

${ }^{46}$ K. Joshi, D. G. Kanhere, and S. A. Blundell, Phys. Rev. B 66, 155329 (2002).

${ }^{47}$ S. Chacko, K. Joshi, and D. G. Kanhere, Phys. Rev. Lett. 92, 135506 (2004).

${ }^{48}$ M. J. Lopez, P. A. Marcos, and J. A. Alonso, J. Chem. Phys. 104, 1056 (1995).

${ }^{49}$ E. Apra, F. Baletto, R. Ferrando, and A. Fortunelli, Phys. Rev. Lett. 93, 065502 (2004). 